\title{
Single-layer versus double-layer closure of the vaginal cuff with barbed sutures in laparoscopic hysterectomy
}

\author{
Dogukan Yildirim ${ }^{1}$, Sefik Eser Ozyurek², Huseyin Kiyak' ${ }^{\text {, Agahan Han }}{ }^{\text {, Nadiye Koroglu }}{ }^{1}$, \\ Aysegul Bestel ${ }^{1}$, Fitnat Topbas ${ }^{1}$ \\ ${ }^{1}$ Department of Obstetrics and Gynecology, Saglik Bilimleri University, Kanuni Sultan Suleyman Training and Research Hospital, \\ Istanbul, Turkey \\ ${ }^{2}$ Department of Obstetrics and Gynecology, Saglik Bilimleri University, Bagcilar Training and Research Hospital, Istanbul, Turkey
}

\begin{abstract}
Objectives: The utilization of barbed sutures in laparoscopic hysterectomy has become popular among gynecologic surgeons. Our aim was to compare the outcomes of two different techniques for closing the vaginal cuff with barbed sutures in laparoscopic hysterectomies.

Material and methods: A retrospective study was completed on 202 patients who underwent laparoscopic hysterectomy for benign diseases at Istanbul Kanuni Sultan Suleyman Training and Research Hospital from April 2014 through June 2016. In group $1(n=139)$, a single-layer continuous suturing method was used; each bite contained the pubocervical fascia and vaginal mucosa anteriorly, and vaginal mucosa and rectovaginal fascia posteriorly. In group $2(n=63)$, a double-layer continuous suturing method was used; only vaginal mucosa was included in the first layer, and a second layer incorporated the pubocervical and rectovaginal fascias.

Results: Patient characteristics (age, body mass index, parity, previous abdominal surgery, smoking, comorbidity) were similar between the two groups. There were also no differences in total operation time, length of hospitalization, intraoperative complications, and perioperative change in hemoglobin levels. There was no difference between the two groups in terms of vaginal cuff dehiscence, which was the primary outcome measure of the study. Secondary outcome measures (presence of granulation tissue, spotting, cuff cellulitis) were also similar between the two groups.

Conclusions: We observed no differences in outcomes between single- or double-layer vaginal closure techniques with barbed sutures.
\end{abstract}

Key words: barbed suture, vaginal cuff dehiscence, laparoscopic hysterectomy, closure technique

Ginekologia Polska 2018; 89, 5: 229-234

\section{INTRODUCTION}

Hysterectomy is one of the most common gynecologic operations in the world. Approximately 600,000 procedures are executed each year in the United States compared with $1,000,000$ cases in China [1]. It can be performed abdominally, vaginally or endoscopically. The American Congress of Obstetricians and Gynecologist recommends using a minimally invasive approach for the benefit of the patients and the associated reduced health care costs [2]. Today, laparoscopic hysterectomy (LH) is considered as a minimally invasive procedure when vaginal hysterectomy is not feasible because of anatomic difficulties.
Reich described the first LH in 1989 [3]. Since then, the trend of using LH has steadily increased from $0.3 \%$ in 1990 to $16.8-24.9 \%$ in $2010[4,5]$. It has been associated with improved outcomes such as decreased morbidity, shorter hospital stay, and quicker return to normal activities when compared with the abdominal approach [6]. However, LH has not been widely used because of its technical difficulties such as intracorporeal suturing of the vaginal vault. With the recent introduction of barbed suture technology, more surgeons are performing laparoscopic cuff suturing without tying knots.

Vaginal cuff dehiscence (VCD) is a partial or total separation of the edges of the vaginal cuff with or without

Corresponding author:

Dogukan Yildirim

Department of Obstetrics and Gynecology, Saglik Bilimleri University, Kanuni Sultan Suleyman Training and Research Hospital, Istanbul, Turkey

e-mail: dogukanyildirim@yahoo.com 
bowel evisceration [7]. This complication is more common with LH when compared with both abdominal and vaginal approaches [8]. The use of barbed sutures decreases the rate of VCD in endoscopic surgery $[9,10]$. These results suggest that barbed sutures are a safe and valuable for vaginal cuff closure. To date, all previous studies compared the results of barbed sutures with conventional sutures. In this retrospective cohort study, however, we only focused on barbed sutures. We compared two different techniques for closing the vaginal vault using barbed sutures among women who underwent LH in our institution; single-layer closure in group 1 and double-layer closure in group 2.

\section{MATERIAL AND METHODS}

Local ethics board approval was granted for the study (Decree no: 624). We performed a retrospective study of patients who underwent laparoscopic hysterectomy for benign indications by a single group of gynecologic surgeons in Istanbul Kanuni Sultan Suleyman Training and Research Hospital between April 2014 and June 2016. All three gynecologists (DY, $\mathrm{HK}, \mathrm{AH}$ ) were experienced in laparoscopic surgery at the beginning of the study. All patient files, which contain operative reports, clinic notes, anesthesia records, discharge summaries, and telephone contacts, were collected from the hospital archive and reviewed. Additional data were retrieved from the electronic medical database system. Patient characteristics (age, body mass index [BMI], obstetric history, surgical history, indication for surgery, comorbidities), operation characteristics (operating time, perioperative blood parameter changes, postoperative hospital stay), and intraoperative and postoperative complications were obtained. All of patients were reexamined between September 2016 and July 2017 to detect any existing vaginal cuff granuloma. During this visit, we also questioned the presence of postoperative complications that might have been managed by another hospital because some patients were not residing in the city where the study was conducted. In the event that such a condition was detected, the related documents and reports were provided and recorded. Patients who could not be contacted or were not willing to participate were excluded from the study. Subjects were also excluded from the analysis if they had an additional urogynecologic procedure, cuff closure by vaginal route or conversion to laparotomy. The primary outcome measure was dehiscence of the vaginal cuff. Secondary outcome parameters were the presence of granulation tissue, postoperative vaginal bleeding (or spotting), and cuff cellulitis.

All patients underwent the same routine preparation before surgery including administration of prophylactic antibiotics. All laparoscopic hysterectomies (LH) were performed in a standard fashion. In brief, our laparoscopic hysterectomy technique was as follows. An intrauterine manipulator
(Clermont-Ferrand, Karl Storz, Tuttlingen, Germany) was inserted into the uterus. After pneumoperitoneum was created, a $10 \mathrm{~mm}$ umbilical trocar port for the camera and three ancillary $5 \mathrm{~mm}$ trocar ports were inserted for instrumentation. Advanced bipolar devices (LigaSure; Valleylab, Inc., Boulder, CO or Enseal; Ethicon Endo-Surgery, Blue Ash, Ohio) were used to seal and transect the ligaments and pedicles. Colpotomy was performed by using monopolar energy with a pure cutting current. If necessary, uterine morcellation was performed vaginally or laparoscopically. After removal of the uterus from the vagina, bipolar energy was used to achieve cuff hemostasis at the lowest level.

Unidirectional barbed sutures $(3-0,15 \mathrm{~cm}$ length, $26 \mathrm{~mm}$ half circle taper point needle, V-Loc ${ }^{\mathrm{TM}}$; Covidien, Mansfield, $M A)$ were used laparoscopically for vaginal cuff closure in all patients. Two surgeons ( $\mathrm{HK}, \mathrm{AH})$ used a single-layer continuous suturing method in group 1; starting from the left corner, each bite contained the pubocervical fascia and vaginal mucosa anteriorly, and vaginal mucosa and rectovaginal fascia posteriorly. The running suture was cut without tying a knot at the right corner (Fig. 1). The other surgeon (DY)_used a double-layer continuous suturing method in group 2; only vaginal mucosa was included in the first layer, and a second layer incorporated the pubocervical and rectovaginal fascias (not vaginal mucosa) to run the suture. The barbed suture was cut without tying a knot at the right corner (Fig. 2). The patients were instructed to abstain from

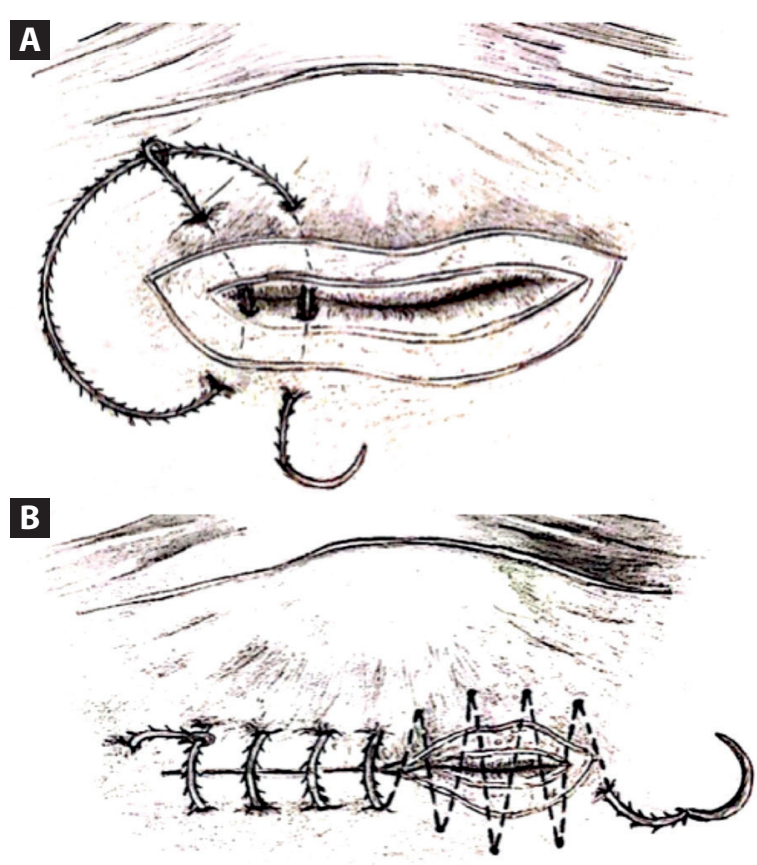

Figure 1. Single-layer suturing method in group 1. A. Suturing was started from the left corner B. Each bite contained the pubocervical fascia and vaginal mucosa anteriorly, and vaginal mucosa and rectovaginal fascia posteriorly 

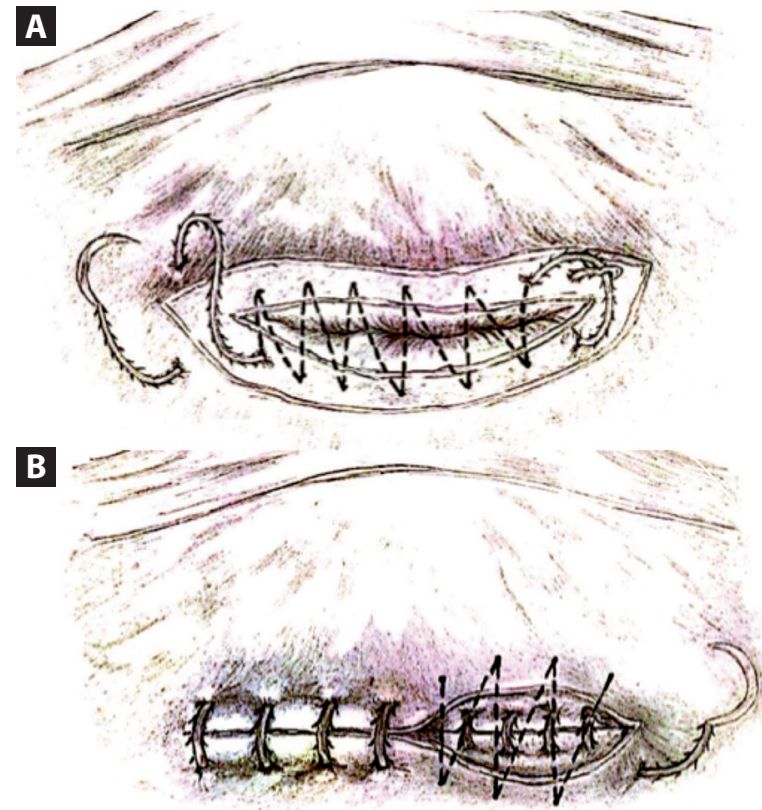

Figure 2. Double-layer suturing method in group 2. A. Suturing was started from the right corner including only the vaginal mucosa in the first layer. B. A second layer incorporated the pubocervical and rectovaginal fascias (not vaginal mucosa)

intercourse and deep bathing for at least eight weeks after the surgery.

The demographic factors and outcome variables were compared among groups 1 and 2 using univariate tests. Parametric tests were used when equal variances and normal distribution could be confirmed. Otherwise, nonparametric tests were used to compare the parameters of the two groups. Significance was considered when $P$ values were lower than 0.05 . The data entry and statistical tests were performed using Microsoft Office 2010 and SPSS 22.0 software.

\section{RESULTS}

The records of 285 patients who underwent total laparoscopic hysterectomy performed by the three surgeons within the annotated time were retrieved from the database system. Among 83 patients who were excluded from the study: 37 had additional urogynecologic procedures, 29 had been sutured with conventional materials, 5 were converted to laparotomy, 9 could not be contacted, and 3 were not willing to participate in the study. A total number of 202 patients were analyzed; 139 (69\%) comprised the single-layer group (group 1), and 63 (31\%) were included in the double-layer group (group 2).

Indications for surgery in group 1 included symptomatic uterine leiomyoma $(n=71)$, adenomyosis-endometriosis $(n=23)$, abnormal uterine bleeding resistant to medical treatment $(n=42)$, and persistent adnexal mass after menopause $(n=3)$. In group 2 , surgical indications were symptomatic uterine leiomyoma $(n=34)$, adenomyosis-endometriosis $(n=9)$, and abnormal uterine bleeding resistant to medical treatment $(n=20)$. Overall, the most frequent indication for surgery was uterine leiomyoma (51\%) and there was no statistically significant difference between the two groups.

Six intraoperative complications occurred; three bladder injuries and two sigmoid serosal lacerations were repaired laparoscopically during the same operation. One omental injury related to the direct primary trocar entry was localized and sealed to stop bleeding. These patients were not excluded from the study because these complications did not relate to the study outcomes.

The results of the comparison performed with univariate analysis stratified by closure technique are shown in Tables 1 and 2 . The mean age of the study population was 49.7 years (range, 35-82 years), and there were no statistical-

Table 1. Clinical features of the groups

\begin{tabular}{|c|c|c|c|}
\hline & $\begin{array}{c}\text { Group } 1 \\
\text { (Single Layer) } \\
n=139\end{array}$ & $\begin{array}{c}\text { Group } 2 \\
\text { (Double Layer) } \\
n=63\end{array}$ & $\mathbf{P}$ \\
\hline Age mean \pm SEM & $49.3(0.63)$ & $50.4(0.66)$ & 0.34 \\
\hline $\mathrm{BMI}$ mean $\pm \mathrm{SEM}$ & $30.8(0.46)$ & $29.8(0.42)$ & 0.19 \\
\hline Parity mean \pm SEM & $2.97(0.12)$ & $2.79(0.15)$ & 0.37 \\
\hline Duration of the operation $(\mathrm{min})$ mean \pm SEM & $138.9(3.5)$ & $141.1(5)$ & 0.74 \\
\hline$\Delta \mathrm{Hb}(\mathrm{g} / \mathrm{dL})$ Mean $\pm \mathrm{SEM}$ & $1.46(0.72)$ & $1.43(0.73)$ & 0.80 \\
\hline Any previous abdominal surgery $\mathrm{n}(\%)$ & $32(23)$ & $19(30.2)$ & 0.30 \\
\hline Diabetes n (\%) & $11(7.9)$ & $6(9.5)$ & 0.79 \\
\hline Hypertension n (\%) & $34(24.5)$ & $12(19)$ & 0.47 \\
\hline Smoking n (\%) & $7(5)$ & $7(11.1)$ & 0.14 \\
\hline Peripheral vascular disease $\mathrm{n}(\%)$ & $3(2.2)$ & $1(1.6)$ & 0.99 \\
\hline Intraoperative complication n (\%) & $4(2.9)$ & $2(3.2)$ & 0.82 \\
\hline
\end{tabular}

SEM — standart error of the mean; BMI — body mass index; $\mathrm{Hb}$ - haemoglobin 
Table 2. Outcome measures of the study groups

\begin{tabular}{|l|c|c|c|}
\hline & $\begin{array}{c}\text { Group 1 } \\
\text { (single layer) } \\
\mathbf{n = 1 3 9}\end{array}$ & $\begin{array}{c}\text { Group 2 } \\
\text { (double layer) } \\
\mathbf{n = 6 3}\end{array}$ & $\mathbf{P}$ \\
\hline Vaginal cuff dehiscence $\mathrm{n}(\%)$ & $1(0.7)$ & $0(0)$ & 0.99 \\
\hline Granuloma tissue $\mathrm{n}(\%)$ & $24(17.3)$ & $6(9.5)$ & 0.20 \\
\hline Spotting $\mathrm{n}(\%)$ & $22(15.8)$ & $9(14.3)$ & 0.83 \\
\hline Cuff cellulitis $\mathrm{n}(\%)$ & $7(5)$ & $3(4.8)$ & 0.99 \\
\hline
\end{tabular}

ly significant differences between the two groups. Twenty-three percent of the patients in the single-layer group had a previous abdominal surgery, as did $30.2 \%$ of patients in the double-layer group; the difference was not statistically significant. The mean duration of the operation was $139 \mathrm{mi}-$ nutes ( \pm 2.86 minutes) in the entire study population and similar between the two groups. No statistically significant differences were found with respect to BMI, parity, change in hemoglobin levels $(\Delta \mathrm{Hb})$, length of hospital stay, smoking and comorbidities (Tab. 1).

The primary outcome measure, VCD, was seen in only one patient in the single-layer group. There was no statistically significant difference between the two groups (Tab. 2). The patient with VCD was aged 40 years with a BMI of 32.9 who underwent total laparoscopic hysterectomy for a symptomatic leiomyoma. Her operation was uneventful and took 90 minutes to perform. She presented with vaginal bleeding 9 days after surgery and reported not having antecedent intercourse. No bowel evisceration was associated; accordingly, she was started on antibiotics and treated expectantly.

The rates of vaginal spotting were similar between the two groups: $15.8 \%$ in the single-layer group and $14.3 \%$ in the double-layer group. No major bleeding occurred in either group apart from in the patient with VCD. Although vaginal cuff granuloma rates were lower in the double-layer group (17.3\% in group 1 vs. 9.5\% in group 2), the difference did not reach a statistically significant level $(p=0.15)$. Seven cases (5\%) in single layer group and 3 cases (4.8\%) in double layer group with cuff cellulitis were treated medically; the difference was not statistically significant.

\section{DISCUSSION}

The findings of this retrospective analysis suggest that single- or double-layer vaginal cuff closure in LH using barbed sutures provide similar results with respect to VCD and also granulation tissue generation, postoperative vaginal bleeding (i.e., spotting) or cuff cellulitis.

With advances in technology, the laparoscopic approach is being increasingly used for total hysterectomy. Several studies demonstrated that laparoscopic hysterectomy (LH) associates with shorter hospital stay, less surgical infection, and faster recovery [5]. Along with the rapid boost in the rate of $\mathrm{LH}$, an increased incidence of vaginal cuff dehiscence (VCD) has been noted. The incidence of this rare complication is not clear and varies between $0 \%$ and $5 \%[11,12]$. In a review, the rate of VCD increased from $0.1 \%$ with abdominal hysterectomy to $5 \%$ with LH [8]. When VCD is associated with bowel evisceration, some complications could occur in the absence of proper management including bowel perforation, peritonitis, and sepsis [13].

Smoking, diabetes, advanced age, immunosuppression, early coital activity, postoperative infections, and cuff hematoma have been associated with an increased risk of VCD $[14,15]$. Nevertheless, the majority of VCD is seen without any detectable cause [8]. It has been postulated that laparoscopic colpotomies using monopolar energy may be an underlying factor due to tissue necrosis and prolonged devascularization [8]. Also, some histopathology studies suggested that the amount and the type of energy used for colpotomy could predispose to VCD $[16,17]$. However, several studies failed to reveal an association between monopolar energy and an increased risk of VCD in laparoscopic hysterectomies $[12,18]$. Given all these considerations, perhaps the causes of VCD dwell in surgical technique, especially in the closing process of the vaginal cuff.

Suturing of the vaginal cuff is a critical component of $\mathrm{LH}$ with many variations in surgical technique and materials. Insufficient suture placement, decreased knot security and suture fraying may have an impact on the development of VCD [7]. In 2007, a revolutionary suture design, the barbed suture, was introduced to the market to facilitate laparoscopic suturing. Barbed sutures do not require an assistant to apply tension to the suture thread, unlike continuous suturing with conventional materials. Moreover, they close the tissue without the use of surgical knots, which is the weakest point of the suture line [19]. In this study, we focused on the performance of barbed sutures by not including cases of vaginal closure with conventional sutures. However, several studies in the literature compared traditional suture materials to barbed sutures.

Only two studies found that barbed sutures decreased the rate of VCD compared with standard suture materials $[9,10]$. On the other hand, many studies could not find any 
association between the two groups for VCD [20-27]. In a meta-analysis, Bogliogo et al. [28] reported that the rate of minor bleeding, major bleeding, and VCD was similar in minimally invasive hysterectomy with or without the use of barbed sutures. They found that barbed sutures reduced the time for vaginal cuff suturing. In a review, Smith et al. [29] also found that barbed sutures decreased the average total procedure time by 15.6 minutes and 5.4 minutes for vaginal cuff suturing. This shortened surgical time may theoretically compensate for the elevated cost of barbed sutures [25]. We did not measure the time for cuff suturing in our study; however, the total operation time was similar in both groups.

Jeung et al. [30] compared the double-layer continuous method with interrupted figure-of-eight sutures using Vicryl in both groups and found no benefits for the double-layer suturing group. Several studies that compared barbed sutures (with double-layer cuff closure) with conventional sutures reported similar results with regard to spotting and cellulitis $[20,21,23,25]$. In contrast, two studies performing a similar comparison found a decrease in the proportion of patients with vaginal bleeding (spotting) using double- layer barbed sutures $[9,22]$. Regarding granuloma, two studies reported that there was no advantage of double-layer closure with barbed sutures [20,22]. Another two studies, however, found less granuloma tissue formation with double-layer barbed sutures $[9,24]$. Our study is the first to compare the double-layer technique with single-layer closure using barbed sutures in both groups. We found no difference between the two groups regarding VCD, cuff granuloma, spotting or cellulitis.

Cuff complications after hysterectomy other than VCD are not rare and can cause discomfort for patients. Bleeding (spotting) is the main reason for repeat consultations in the postoperative period after $\mathrm{LH}$ [22]. We found that $15.3 \%$ of the entire patient population experienced spotting; it was the most common complication of our study. We could not measure the duration of spotting due to the retrospective design of our study. We observed that some patients reported prolonged periods of spotting of up to two months. Vaginal cuff granuloma was also frequent (14.8\%) in our patient population and was even defined beyond two years following the operation in individual cases. One could presume that there might be an association between spotting and granuloma. However, only 7 of 30 patients (23.3\%) with cuff granuloma reported spotting in our study, with no significant association.

Our institution has been using barbed sutures for vaginal cuff closure in laparoscopic hysterectomies since 2013. Although some surgeons still prefer to use conventional braided sutures, barbed sutures became quite popular in our hospital. We only included the patients of three surgeons because these surgeons performed a high volume of LH within the defined period, thereby eliminating the influence of experience variability originating from different surgeons being at different points in their learning curves in LH. The retrospective design was a limitation of our study. Therefore, the allocation of cases to the two groups was not randomized due to which the group sizes were uneven. Moreover, it was possible to have recall bias because some patients may not have remembered all the potential symptoms during the early postoperative period (e.g., spotting). Another limitation was the rarity of the primary outcome (VCD), which makes it difficult to demonstrate a decrease in the incidence of this event in a relatively small study population.

\section{CONCLUSIONS}

We observed no differences in outcomes between single- or double-layer vaginal closure techniques with barbed sutures. The preferred method, whether single- or double-layer closure of the vaginal cuff in LH, should be founded on the surgeon's choice. This issue merits randomized controlled prospective trials.

\section{Conflict of interest}

The authors declare that they have no conflict of interest.

\section{Acknowledgements}

We thank Dr Seyma Yesiralioglu for the illustrations.

\section{REFERENCES}

1. Cong L, Li C, Wei B, et al. V-Loc ${ }^{\mathrm{TM}} 180$ suture in total laparoscopic hysterectomy: a retrospective study comparing Polysorb to barbed suture used for vaginal cuff closure. Eur J Obstet Gynecol Reprod Biol. 2016; 207: 18-22, doi: 10.1016/j.ejogrb.2016.09.012, indexed in Pubmed: 27816737.

2. ACOG Committee Opinion No. 444: choosing the route of hysterectomy for benign disease. Obstet Gynecol. 2009; 114(5): 1156-1158, doi: 10.1097/AOG.0b013e3181c33c72, indexed in Pubmed: 20168127.

3. REICH H, DeCAPRIO J, McGLYNN F. Laparoscopic Hysterectomy. Journal of Gynecologic Surgery. 1989; 5(2): 213-216, doi: 10.1089/gyn.1989.5.213.

4. Cohen SL, Vitonis AF, Einarsson Jl. Updated hysterectomy surveillance and factors associated with minimally invasive hysterectomy. JSLS. 2014; 18(3), doi: 10.4293/JSLS.2014.00096, indexed in Pubmed: 25392662.

5. Jacoby VL, Autry A, Jacobson G, et al. Nationwide use of laparoscopic hysterectomy compared with abdominal and vaginal approaches. Obstet Gynecol. 2009; 114(5): 1041-1048, doi: 10.1097/AOG.0b013e3181b9d222, indexed in Pubmed: 20168105.

6. Desai VB, Guo XM, Fan L, et al. Inpatient Laparoscopic Hysterectomy in the United States: Trends and Factors Associated With Approach Selection. J Minim Invasive Gynecol. 2017; 24(1): 151-158.e1, doi: 10.1016/j. jmig.2016.08.830, indexed in Pubmed: 27614151.

7. Fanning J, Kesterson J, Davies M, et al. Effects of electrosurgery and vaginal closure technique on postoperative vaginal cuff dehiscence. JSLS. 2013; 17(3): 414-417, doi: 10.4293/10860813X1369342251851, indexed in Pubmed: 24018078.

8. Hur HC, Guido RS, Mansuria SM, et al. Incidence and patient characteristics of vaginal cuff dehiscence after different modes of hysterectomies. J Minim Invasive Gynecol. 2007; 14(3): 311-317, doi: 10.1016/j. jmig.2006.11.005, indexed in Pubmed: 17478361.

9. Siedhoff MT, Yunker AC, Steege JF. Decreased incidence of vaginal cuff dehiscence after laparoscopic closure with bidirectional barbed suture. J Minim Invasive Gynecol. 2011; 18(2): 218-223, doi: 10.1016/j. jmig.2011.01.002, indexed in Pubmed: 21354068.

10. Rettenmaier MA, Abaid LN, Brown JV, et al. Dramatically reduced incidence of vaginal cuff dehiscence in gynecologic patients undergoing endoscopic closure with barbed sutures: A retrospective cohort study. Int J Surg. 2015; 19: 27-30, doi: 10.1016/j.jisu.2015.05.007, indexed in Pubmed: 25980394 
11. Nieboer TE, Johnson N, Lethaby A, et al. Surgical approach to hysterectomy for benign gynaecological disease. Cochrane Database Syst Rev. 2009(3): CD003677, doi: 10.1002/14651858.CD003677.pub4, indexed in Pubmed: 19588344

12. Uccella $S$, Ceccaroni M, Cromi A, et al. Vaginal cuff dehiscence in a series of 12,398 hysterectomies: effect of different types of colpotomy and vaginal closure. Obstet Gynecol. 2012; 120(3): 516-523, doi: 10.1097/AOG.0b013e318264f848, indexed in Pubmed: 22914459.

13. Ceccaroni $M$, Berretta $R$, Malzoni M, et al. Vaginal cuff dehiscence after hysterectomy: a multicenter retrospective study. Eur J Obstet Gynecol Reprod Biol. 2011; 158(2): 308-313, doi: 10.1016/j.ejogrb.2011.05.013, indexed in Pubmed: 21723030

14. Hur HC, Donnellan N, Mansuria S, et al. Vaginal cuff dehiscence after different modes of hysterectomy. Obstet Gynecol. 2011; 118(4): 794-801, doi: 10.1097/AOG.0b013e31822f1c92, indexed in Pubmed: 21934442.

15. Somkuti SG, Vieta PA, Daugherty JF, et al. Transvaginal evisceration after hysterectomy in premenopausal women: a presentation of three cases. Am J Obstet Gynecol. 1994; 171 (2):567-568, indexed in Pubmed: 8059847.

16. Tulikangas PK, Smith T, Falcone T, et al. Gross and histologic characteristics of laparoscopic injuries with four different energy sources. Fertil Steril. 2001; 75(4): 806-810, indexed in Pubmed: 11287039.

17. Gruber DD, WarnerWB, Lombardini ED, et al. Laparoscopic hysterectomy using various energy sources in swine: a histopathologic assessment. Am J Obstet Gynecol. 2011; 205(5): 494.e1-494.e6, doi: 10.1016/j. ajog.2011.07.009, indexed in Pubmed: 21924395.

18. Uccella S, Ghezzi F, Mariani A, et al. Vaginal cuff closure after minimally invasive hysterectomy: our experience and systematic review of the literature. Am J Obstet Gynecol. 2011; 205(2): 119.e1-119.12, doi: 10.1016/j. ajog.2011.03.024, indexed in Pubmed: 21620360.

19. Greenberg JA. The use of barbed sutures in obstetrics and gynecology. Rev Obstet Gynecol. 2010; 3(3): 82-91, indexed in Pubmed: 21364859.

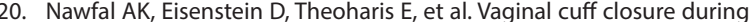
robotic-assisted total laparoscopic hysterectomy: comparing vicryl to barbed sutures. JSLS. 2012; 16(4): 525-529, doi: 10.4293/108680812X1 3462882736772, indexed in Pubmed: 23484558.

21. Ardovino M, Castaldi MA, Fraternali $F$, et al. Bidirectional barbed suture in total laparoscopic hysterectomy and lymph node dissection for endometrial cancer: technical evaluation and 1-year follow-up of 61 patients. J Laparoendosc Adv Surg Tech A. 2013; 23(4): 347-350, doi: 10.1089/lap.2012.0079, indexed in Pubmed: 23573880.

22. Medina $B C$, Giraldo $\mathrm{CH}$, Riaño $\mathrm{G}$, et al. Barbed suture for vaginal cuff closure in laparoscopic hysterectomy. JSLS. 2014; 18(1): 83-88, doi: 10. 4293/108680813X13693422518795, indexed in Pubmed: 24680149.

23. Kim JH, Byun SW, Song JY, et al. Barbed versus conventional 2-layer continuous running sutures for laparoscopic vaginal cuff closure. Medicine (Baltimore). 2016; 95(39): e4981, doi: 10.1097/MD.0000000000004981, indexed in Pubmed: 27684850.

24. Cong L, Li C, Wei B, et al. V-Loc ${ }^{\mathrm{TM}} 180$ suture in total laparoscopic hysterectomy: a retrospective study comparing Polysorb to barbed suture used for vaginal cuff closure. Eur J Obstet Gynecol Reprod Biol. 2016; 207: 18-22, doi: 10.1016/j.ejogrb.2016.09.012, indexed in Pubmed: 27816737.

25. Tsafrir Z, Palmer M, Dahlman M, et al. Long-term outcomes for different vaginal cuff closure techniques in robotic-assisted laparoscopic hysterectomy: A randomized controlled trial. Eur J Obstet Gynecol Reprod Biol. 2017; 210: 7-12, doi: 10.1016/j.ejogrb.2016.11.018, indexed in Pubmed: 27923167.

26. Blikkendaal MD, Twijnstra $A R H$, Pacquee $S C L$, et al. Vaginal cuff dehiscence in laparoscopic hysterectomy: influence of various suturing methods of the vaginal vault. Gynecol Surg. 2012; 9(4): 393-400, doi: 10.1007/s10397-012-0745-5, indexed in Pubmed: 23144640.

27. Einarsson Jl, Cohen SL, Gobern JM, et al. Barbed versus standard suture: a randomized trial for laparoscopic vaginal cuff closure. J Minim Invasive Gynecol. 2013; 20(4): 492-498, doi: 10.1016/j.jmig.2013.02.015, indexed in Pubmed: 23680517

28. Bogliolo $S$, Nadalini $C$, lacobone $A D$, et al. Vaginal cuff closure with absorbable bidirectional barbed suture during total laparoscopic hysterectomy. Eur J Obstet Gynecol Reprod Biol. 2013; 170(1): 219-221, doi: 10.1016/j.ejogrb.2013.06.006, indexed in Pubmed: 23820139.

29. Smith K, Caceres A. Vaginal Cuff Closure in Minimally Invasive Hysterectomy: A Review of Training, Techniques, and Materials. Cureus. 2017; 9(10): e1766, doi: 10.7759/cureus.1766, indexed in Pubmed: 29234570.

30. Jeung InC, Baek JM, Park EK, et al. A prospective comparison of vaginal stump suturing techniques during total laparoscopic hysterectomy. Arch Gynecol Obstet. 2010; 282(6): 631-638, doi: 10.1007/s00404-009-1300-0, indexed in Pubmed: 19943055. 